\title{
Estudio preliminar del ajuste entre datos meteorológicos in situ y del Reanálisis (NCEP/NCAR) en distintos ambientes de la provincia de Buenos Aires, Argentina
}

\section{INTRODUCCIÓN}

En las últimas décadas se ha extendido el uso de modelos numéricos para el estudio de parámetros climáticos. Uno de los más utilizados es el Reanálisis (NCEP/NCAR $)^{1}$. El mismo se ha empleado para el estudio del clima mundial y regional ${ }^{2}$. A menor escala espacial se encuentran, por ejemplo, los trabajos de Guedes et al. ${ }^{3}$ que analizaron los datos de precipitación del Reanálisis para la localidad de Fortaleza (Brasil) obteniendo una buena concordancia entre

${ }^{1}$ El Reanálisis (NCEP/NCAR) es un modelo numérico que permite obtener información meteorológica mensual de diversos elementos del clima (temperatura, humedad relativa, precipitación, presión atmosférica, etc.) desde 1948 a la actualidad y poseen cobertura mundial. Son gratuitos y de libre acceso (http://www.esrl.noaa.gov/psd/data/reanalysis).

${ }^{2}$ Kalnay, E., Kanamitsu, M., Kistler, R., Collins, W., Deaven, D., Gandin, L., Iredell, M., Saha, S., White, G., Woolten, J., Zhu, Y., Chellian, M., Ebisuzaki, W., Higgins, W., Janowiak, J., Mo, K. C., Ropelewski, C., Wang, J., Leetmaa, A., Reynolds, R., Jene, R. y Joseph, D. (1996): "The NCEP/ NCAR 40-year Reanalysis Project", Bulletin of the American Meteorological Society, 77, pp. 437-471. Klister, R., Kalnay, E., Collins, W., Saha S., White, G., Woollen, J., Chelliah, M., Ebisuzaki, W., Kanamitsu, M., Kousky, V., Van del Dool, H., Jenne, R. y Fiorino, M. (2001): "The NCEP NCAR 50 year Reanalysis: Monthly Means CD-ROM and documentation", Bulletin of the American Meteorological Society, 81, pp. 247-267. Rusticucci, M. M. y Kousky V. E. (2002): "A comparative study of maximum and minimum temperatures over Argentina: NCEPNCAR Reanalysis versus station data”, American Meterological Society, 15, pp. 2089-2101.

${ }^{3}$ Simmons, A. J., Jones, P. D., da Costa Bechtold, V., Beljaars, A. C. M., Kallberg, P., Saarinen, W. S., Uppala, S. M., Viterbo, P. y Wedi, N. (2004): "Comparison of trends and low-frequency variability in CRU, ERA-40, and NCEP/NCAR analyses of surface air temperature", Journal of Geophysical Research, 109, pp. 1-18. 
ellos y los datos in situ. Pescio et al. ${ }^{4}$ implementaron el modelo Simulating WAves Nearshore (SWAN) para realizar una simulación de 35 años de la altura significativa de las olas en el área del estuario de Bahía Blanca, utilizando datos de viento del NCEP/NCAR.

El área de estudio de este trabajo es el SW de la provincia de Buenos Aires (figura 1). Su economía se basa en la producción agropecuaria y el turismo, los cuales dependen en gran medida de las características climáticas y meteorológicas. El problema general del área es la falta de datos meteorológicos históricos continuos que permitan realizar estimaciones meteorológicas para sustentar los calendarios agrícolas y turísticos. Por ellos, el objetivo del presente trabajo fue analizar en forma preliminar el ajuste entre los datos del Reanálisis (NCEP/NCAR) y los datos in situ en distintos ambientes localizados dentro de una retícula del modelo $(278,3 \mathrm{~km}$ por $278,3 \mathrm{~km})$ con el fin de obtener datos meteorológicos.

\section{Área de estudio}

El área de estudio se encuentra dentro del clima templado subhúmedo (por sus escasas precipitaciones, $500 \mathrm{~mm}$ ) o templado de transición (por sus rasgos de aridez y su transición hacia la región patagónica). El mismo se caracteriza por la influencia de diferentes masas de aire que modifican constantemente sus condiciones del tiempo 67 .

Se observan variaciones espaciales en la distribución de la temperatura. Campo et al. ${ }^{8}$ asocian estas diferencias a la presencia de factores locales, entre ellos la presencia del Sistema de Ventania (figura 1). En esta área se encuentran

\footnotetext{
${ }^{4}$ Pescio, A., Martin, P. y Dragani, W. (2012): "Altura de Ola Extrema en la Zona Exterior del Estuario de Bahía Blanca, Pcia. de Buenos Aires, Argentina”, en Actas del 1er Encuentro de Investigadores en Formación en Recursos Hídricos (IFRH 2012), Ezeiza, Buenos Aires, Argentina, Instituto Nacional del Agua.

${ }^{5}$ Chiozza, E. (1975): El país de los argentinos. La Pampa Surera, Buenos Aires, Centro Editor de América Latina S.A. Fascículo $n^{\circ} 42$.

${ }^{6}$ Díaz, R. A. y Mormeneo, I. (2002): "Zonificación del clima de la región pampeana mediante análisis de conglomerados por consenso", Revista Argentina de Agrometeorología, 2/2, pp. 125-131.

${ }^{7}$ Capelli de Steffens, A. y Campo de Ferreras, A. (1994): La transición climática en el Sudoeste Bonaerense, Bahía Blanca, Sección de Investigaciones del Departamento de Geografía, 77 pp. Colección Sigeo, 5.

${ }^{8}$ Campo, A., Capelli de Steffens, A y Diez, P. (2004): El clima del Sudoeste Bonaerense, Bahía Blanca, Departamento de Geografía y Turismo, Universidad Nacional del Sur, 99 pp.
} 


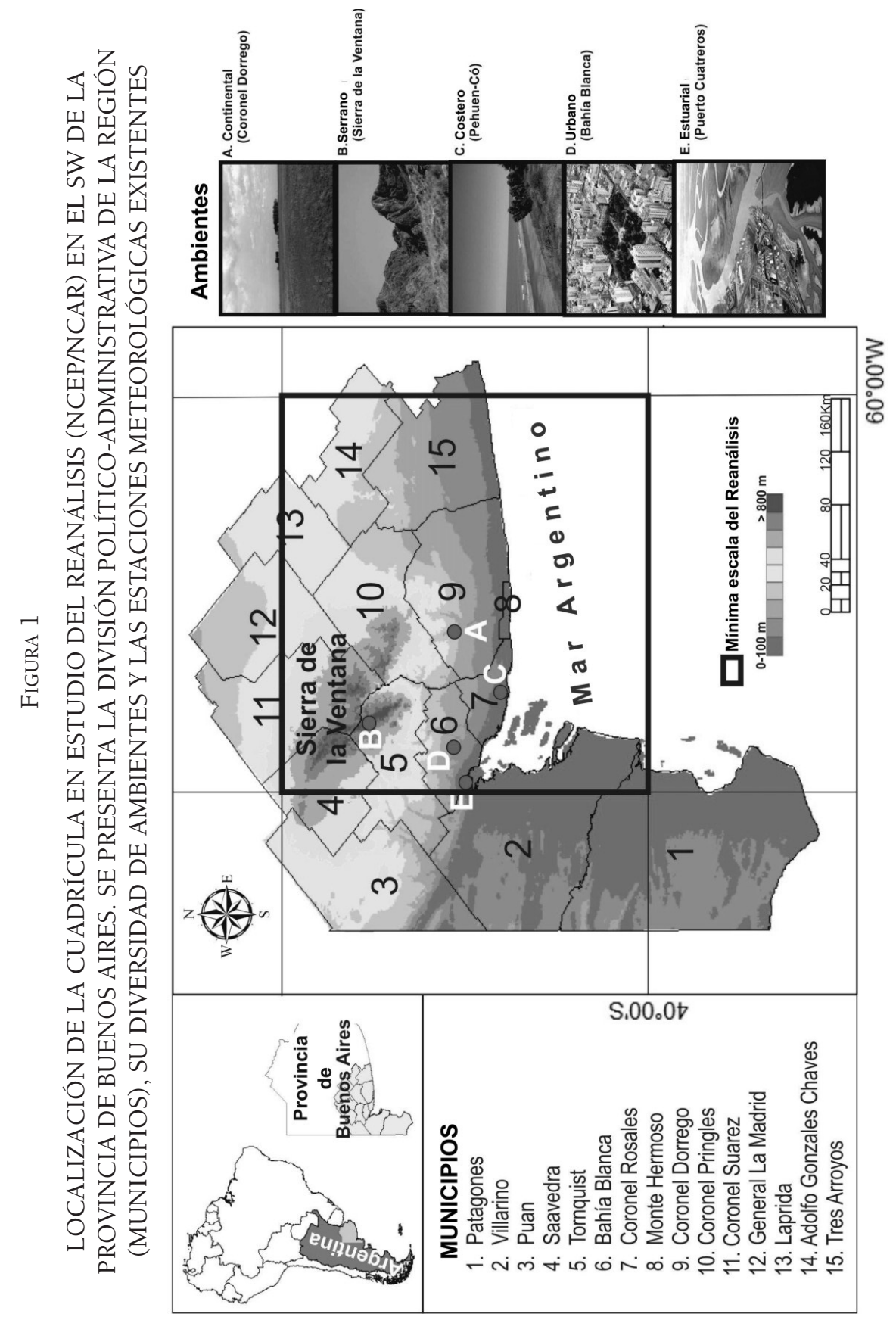

Estudios Geográficos, Vol. LXXVII, 280, pp. 335-343, enero-junio 2016 ISSN: 0014-1496, eISSN: 1988-8546 
las temperaturas medias más bajas de la región $\left(13,4^{\circ} \mathrm{C}\right)$. En dirección al $\mathrm{S}$, los valores térmicos comienzan a incrementarse y llegan en promedio a $14,5^{\circ} \mathrm{C}$. La dirección predominante del viento es del N y NW. Las intensidades en la velocidad del viento aumentan a medida que se aleja de la zona serrana (Sistema de Ventania) ya que esta ofrece una protección ante la acción de los vientos ${ }^{9}$.

\section{MATERIALES Y MÉTODOS}

Se utilizaron datos del modelo numérico "NCEP/NCAR Reanalysis I" (de ahora en más Reanálisis) desarrollado por el Centro Nacional de Predicción Ambiental (NCEP), el Centro Nacional de Investigación Atmosférica (NCAR) y de la National Oceanic and Atmospheric Administration / Climate Diagnostics Center (NOAA / CDC) ${ }^{10}$. Los mismos se encuentran disponibles a nivel mundial en retículas uniformes de 2.5 grados de latitud por 2.5 grados de longitud $(278,3 \mathrm{~km}$ por $278,3 \mathrm{~km})$.

Se escogió una cuadrícula del Reanálisis que comprende diferentes ambientes del área de estudio para determinar a cuáles de ellos se ajustan mejor los datos del modelo numérico (figura 1). Se seleccionaron las siguientes variables: temperatura del aire, humedad relativa y precipitación dado que son las más frecuentemente utilizadas en el estudio de la variabilidad climática.

Los ambientes seleccionados fueron (figura 1): 1) Continental: Es un área de topografía llana, con una suave ondulación hacia el $\mathrm{W}$ y pendiente moderada hacia al marli; 2) Serrano: Se extiende por 170 km en dirección NW-SE. Está compuesto por 5 cordones principales con una altura máxima de $1.243 \mathrm{msnm}^{12}$; 3) Costero: incluye playas de mareas semidiurnas, con suave pendiente, arenas de tamaño medio y enmarcadas por médanos móviles y fijos por vegetación ${ }^{13}$;

${ }^{9}$ Campo de Ferreras, A. M., Hernández Moresino, S. D., Huamantinco Cisneros, M. A. y Zapperi, P. A. (2004): "Ocurrencia de sudestadas en el suroeste bonaerense", en Contribuciones Científicas, Santa Fe, Sociedad Argentina de Estudios Geográficos, pp. 45-50.

${ }^{10} \mathrm{http}: / /$ wesley.wwb.noaa.gov/Reanalysis.html

11 Petracci, P. F. (2011): “¿Puede el pisoteo de los Cauquenes (Chloephaga spp.) ocasionar compactación del suelo en cultivos de trigo (triticum aestivum)?”, Hornero, 26/2, pp. 95-103.

${ }^{12}$ Lizzi, J. M., Garbulsky, M. F., Golluscio, R. A. y Deregibus, A. V. (2007): "Mapeo indirecto de la vegetación de Sierra de la Ventana, provincia de Buenos Aires", Ecología austral [online], 17/2, pp. 217-230.

${ }^{13}$ Bustos, M. L., Piccolo, M. C. y Perillo, G. M. E. (2011): "Efectos geomorfológicos de fuertes vientos sobre playas. El caso de la playa de Pehuén Co, Argentina", Cuadernos de Investiga- 
4) Urbano: Posee varias localidades que en general no superan los 10.000 habitantes, solo un área con más de 300.000 habitantes (Bahía Blanca) a la cual se suma una zona industrial y portuaria ${ }^{14}$; y 5) Estuarial: El estuario de Bahía Blanca es un sistema mesomareal formado por una serie de canales principales con dirección NW a SE, poseen una extensión total de $60 \mathrm{~km}$ y un ancho de más de $3 \mathrm{~km}$ en la boca. La zona intermareal se extiende por más de $1 \mathrm{~km}$ a través del gradiente mareal ${ }^{15}$.

Debido a la falta de estaciones meteorológicas y series de datos históricos, se utilizaron sólo datos de 5 estaciones meteorológicas ${ }^{16}$ para el período 2010-2012 (figura 1). Los mismos se promediaron de forma mensual, estacional y anual.

Los datos se analizaron y relacionaron en dos etapas. La primera de ellas incluyó el cálculo del índice de concordancia (C), el coeficiente de determinación $\left(\mathrm{R}^{2}\right)$ y el coeficiente de correlación de Pearson (P). Mientras que en la segunda, el ajuste entre ambas fuentes de datos se efectuó mediante: un análisis de regresión, un análisis de la Varianza y un test de comparación de medias de DMS-Fisher (Diferencia Mínima Significativa) con un de 0,05. La utilización de este último tiene relación con la fácil aplicación del test y con la posibilidad de generar todas las comparaciones con el mismo valor de. Esto permite que los resultados generados con este método sean comparables entre sí debido a que el número de réplicas utilizado en cada comparación fue el mismo para todas las variables consideradas. Su aplicación permitió establecer las diferencias y las igualdades entre las medias anuales de ambas fuentes de información (Reanálisis e in situ) en los distintos ambientes considerados.

ción Geográfica, 37/1, pp. 121-142. Huamantinco Cisneros, M. A. y Piccolo, M. C. (2011): "Caracterización de la brisa de mar en el balneario de Monte Hermoso, Argentina", Estudios Geográficos, LXXII, pp. 461-475.

${ }^{14}$ Piccolo, M. C. y Diez, P. (2008): "Cold and heat impact on human comfort in the Bahía Blanca estuary harbours (Argentina)”, Cuadernos de Investigación Geográfica, 34, pp. 119-131.

${ }^{15}$ Perillo, G. M. E. y Piccolo, M. C. (2004): "Qué es el estuario de Bahía Blanca?" Ciencia Hoy 14/81, pp. 8-15. http://www.ciencia-hoy.retina.ar.

${ }^{16}$ Las estaciones meteorológicas pertenecen a la red de Estaciones de Monitoreo Ambiental Costero (EMAC) (http://emac.criba.edu.ar/) del Instituto Argentino de Oceanografía, al Instituto Nacional de Tecnología Agropecuaria (www.inta.gob.ar) y al Departamento de Geografía y Turismo de la Universidad Nacional del Sur. 
RESUlTAdos

La temperatura presentó mejor ajuste en los ambientes urbano y estuarial, siendo menor en la zona costera y las sierras. El $\mathrm{R}^{2}$ fue mayor a 0,93 en todos los casos estudiados, al igual que los índices de correlación. Esto permitió inferir que existe un buen ajuste de la temperatura en todos los ambientes considerados. A pesar de ello, la concordancia fue menor en el ambiente costero $(0,72)$ y mayor en el continental $(0,95)$. Por otro lado, la temperatura del aire presentó una menor diferencia entre los valores medios anuales en los ambientes serrano $\left(-0,3^{\circ} \mathrm{C}\right)$ y urbano $\left(1^{\circ} \mathrm{C}\right)$, mientras que el mayor contraste se observó en el costero $\left(4,5^{\circ} \mathrm{C}\right)$. El análisis de regresión realizado resultó altamente significativo ( $p$ value $=2,12 \mathrm{E}^{-07}$ ). La temperatura presentó una pendiente de 0,95 y un error típico de 1,35 (tablas 1 y 2 ).

\section{TABLA 1}

PARÁMETROS DE REGRESIÓN PARA LA TEMPERATURA DEL AIRE, HUMEDAD RELATIVA Y PRECIPITACIONES DE LOS DISTINTOS AMBIENTES CONSIDERADOS

\begin{tabular}{l|ccc|ccc|ccc}
\hline Ambientes & \multicolumn{3}{|c|}{ Temperatura del aire } & \multicolumn{3}{c|}{ Humedad relativa } & \multicolumn{3}{|c}{ Precipitación } \\
\hline & $\mathrm{R} 2$ & $\mathrm{P}$ & $\mathrm{C}$ & $\mathrm{R} 2$ & $\mathrm{P}$ & $\mathrm{C}$ & $\mathrm{R} 2$ & $\mathrm{P}$ & $\mathrm{C}$ \\
\hline Urbano & 0.99 & 0.99 & 0.98 & 0.84 & 0.91 & 0.63 & 0.38 & 0.62 & 0.52 \\
Estuarial & 0.99 & 0.99 & 0.96 & 0.83 & 0.91 & 0.31 & 0.19 & 0.43 & 0.26 \\
Continental & 0.98 & 0.99 & 0.95 & 0.81 & 0.78 & 0.27 & 0.78 & 0.88 & 0.80 \\
Costero & 0.95 & 0.98 & 0.71 & 0.73 & 0.21 & 0.15 & 0.14 & -0.37 & -0.11 \\
Serrano & 0.94 & 0.97 & 0.97 & 0.01 & 0.06 & 0.03 & 0.01 & 0.10 & 0.09 \\
\hline
\end{tabular}

$\mathbf{R}^{2}$ Coeficiente de determinación, P Coeficiente de Pearson y C Concordancia.

La humedad relativa mostró mayores diferencias. El mejor ajuste se observó, al igual que en la temperatura, en el ambiente urbano y el menor fue en el ambiente costero. Los valores de $\mathrm{P}$ y $\mathrm{C}$ para los ambientes estuarial y costero fueron menores a 0,4 debido a que el ajuste entre las variables consideradas no fue lineal. A pesar de ello, el R2 calculado siempre fue mayor a 0,7. Los datos del Reanálisis no pueden ser aplicados en la zona serrana dado que no existe correlación (tabla 1). La humedad relativa manifestó mayor desigualdad en el 
continental (15\%) y en el serrano (12\%), aunque estos valores son del mismo orden de magnitud del error de medición del instrumental. También manifestó la menor diferencia entre las medias observadas en los ambientes costero (7\%) y urbano (8\%) (tabla 2). La pendiente fue de 0,94 y el error típico de 3,43. El análisis de regresión fue altamente significativo ( $\mathrm{p}$ value $=3,11 \mathrm{E}-05$ ).

La precipitación mantuvo, en general, un mal ajuste lineal en todos los ambientes. El mayor $\mathrm{R}^{2}$ se observó en el área continental $\left(\mathrm{R}^{2}=0,78\right)$ y no existió correlación en el área serrana. Los coeficientes de correlación se mantuvieron menores a 0,43 en todos los ambientes excepto en el urbano (tabla 1). Los mayores valores de $\mathrm{P}$ y $\mathrm{C}$ se presentaron en el continental con 0,88 y 0,8 ; respectivamente. La precipitación no presentó un buen ajuste en ninguno de los ambientes (tabla 2).

TABLA 2

DIFERENCIA ENTRE LOS VALORES MEDIOS ANUALES DE LOS PARÁMETROS ESTIMADOS DEL REANÁLISIS Y LOS MEDIDOS IN SITU

\begin{tabular}{lccc}
\hline \multicolumn{1}{c}{ Ambientes } & $\mathrm{T}\left({ }^{\circ} \mathrm{C}\right)$ & $\mathrm{HR}(\%)$ & $\mathrm{P}(\mathrm{mm})$ \\
\hline Urbano & 1,0 & 8 & 20,1 \\
Estuarial & 1,5 & 18 & 41,6 \\
Continental & 2,0 & 15 & 11,6 \\
Costero & 4,5 & 7 & 25,7 \\
Serrano & $-0,3$ & 12 & 16,0 \\
\hline
\end{tabular}

T. Temperatura. RH. Humedad Relativa. P. Precipitaciones.

\section{CONCLUSIONES Y DISCUSIÓN}

La temperatura manifestó el mejor ajuste entre los valores in situ y los del Reanálisis, mientras que la precipitación fue el parámetro con menor ajuste seguido por la humedad relativa.

A partir de este análisis preliminar se sugiere que los datos del Reanálisis podrían ser utilizados, principalmente, para la caracterización climática de ambientes urbanos de escasa altitud, como por ejemplo la ciudad de Bahía Blanca. En cambio, la influencia del mar en el ambiente costero provocó que los datos obtenidos por el Reanálisis para la temperatura y precipitación no 
coincidieran con los medidos in situ, tal como sucedió en la investigación de De Ruyver et al. ${ }^{17}$. Respecto a las precipitaciones, se observó un mejor ajuste entre los datos del Reanálisis y los in situ en los ambientes urbano y continental. Tal como afirman Betts et al. ${ }^{18}$ y Guedes et al. ${ }^{19}$ los datos de precipitaciones del NCEP/NCAR se ajustan a las tendencias anuales pero muestran diferencias en los datos mensuales.

En los ambientes serranos se observó que la temperatura del aire tiene un mejor ajuste que los otros parámetros considerados. Esto muestra una diferencia con otros autores que encontraron que a mayor altura sobre el nivel medio del mar menor es el ajuste de los datos observados con los del NCEP/NCAR 20.

Por otro lado, el ambiente urbano presentó un buen ajuste para la temperatura del aire. En este caso, la diferencia entre los valores medios fue de $1{ }^{\circ} \mathrm{C}$ pero el test de Fisher realizado no encontró evidencia de que las medias de ambas fuentes de información fueran estadísticamente distintas. La humedad relativa manifestó los mejores ajustes solo en el ambiente urbano, aunque se observaron diferencias del orden del 8\%, dependiendo del ambiente. Estudios como el de Trenberth y Guillemot ${ }^{21}$ o el de Bao y Zhang ${ }^{22}$, confirman la existencia de diferencias entre los valores medios de la humedad relativa del Reanálisis y los datos observados.

17 De Ruyver, R., Denegri, M. J. y Oricchio, P. (2012): "Mapa de riesgos de temperaturas frías para el sur de la provincia de Buenos Aires usando datos satelitales y de superficie", Revista Meteorológica, 37, pp. 37-46.

18 Betts, A., Hong, S. y Pan, H. (1996): "Comparison of NCEP-NCAR Reanalysis with 1987 FIFE Data”, Monthly Weather Review, 124, pp. 1480-1498.

19 Guedes, R. L., Andreoli, R. V., Kayano, M. T., Oyama, M. D. y Senaubar Alves, M. A., (2005): "Série temporal de precipitação mensal de Fortaleza, Brasil: comparação entre observações e dados de reanálise do NCEP/NCAR", Revista Brasileira de Meteorología, 20/1, pp. 83-92.

${ }^{20}$ Rusticucci, M. M. y Kousky, V. E. (2002): "A comparative study of maximum and minimum temperatures over Argentina: NCEP-NCAR Reanalysis versus station data”, American Meterological Society, 15, pp. 2089-2101. You, Q., Fraedrich, K., Ren, G., Pepin, N. y Kang, S. (2013): "Variability of temperature in the Tibetan Plateau based on homogenized surface stations and reanalysis data", Journal of Climatology, 33, pp. 1337-1347. Bao, X. y Zhang, F. (2013): "Evaluation of NCEP-CFSR, NCEP-NCAR, ERA-Interim, and ERA-40 Reanalysis Datasets against Independent Sounding Observations over the Tibetan Plateau", Journal of Climate, 26, pp. 206-214.

${ }^{21}$ Trenberth, K. E. y Guillemot, C. J. (1998): "Evaluation of the atmospheric moisture and hydrological cycle in the NCEP/NCAR reanalyses", Climate Dynamics, 14, pp. 213-231.

22 Bao, X. y Zhang, F. (2013): "Evaluation of NCEP-CFSR, NCEP-NCAR, ERA-Interim, and ERA-40 Reanalysis Datasets against Independent Sounding Observations over the Tibetan Plateau", Journal of Climate, 26, pp. 206-214. 
La precipitación estimada por el Reanálisis es un parámetro que debería ser corregido dado que no presentó una relación lineal con los datos observados en los ambientes considerados. Guedes et al. ${ }^{23}$ sugieren utilizar estos datos con precaución debido a que si bien los parámetros de correlación pueden ser superiores al 50\% en las medias de más de 20 años, los datos mensuales y anuales difieren de los datos observados.

A pesar de que se reconoce que este estudio analiza un período corto de tiempo, se entiende que este trabajo representa un análisis preliminar que sirve de base para interpretar la información del Reanálisis. La escasez de datos meteorológicos in situ necesita de alternativas que sean viables para realizar una caracterización climática. El SW de la provincia de Buenos Aires posee escaso número de estaciones meteorológicas con largas series de tiempo, por lo que el conocimiento y la interpretación de datos climáticos de otras fuentes de información son herramientas fundamentales para el conocimiento del clima regional y local. Por lo tanto, el mismo adquiere interés como punto de partida para el análisis de datos in situ y su relación con los del Reanálisis con el fin de conocer las características climáticas de distintos ambientes de latitudes medias ${ }^{24}$.

\author{
María Luján Bustos y Federico Ferrelli \\ Universidad Nacional del Sur e Instituto Argentino \\ de Oceanografía (CONICET), Argentina \\ María Andrea Huamantinco Cisneros \\ Instituto Argentino de Oceanografía (CONICET), Argentina \\ María Cintia Piccolo \\ Universidad Nacional del Sur e Instituto Argentino \\ de Oceanografía (CONICET), Argentina \\ Verónica Gil \\ Universidad Nacional del Sur, Argentina
}

${ }^{23}$ Guedes, R. L., Andreoli, R. V., Kayano, M. T., Oyama, M. D. y Senaubar Alves, M. A. (2005): "Série temporal de precipitação mensal de Fortaleza, Brasil: comparação entre observações e dados de reanálise do NCEP/NCAR", Revista Brasileira de Meteorología, 20/1, pp. 83-92.

${ }^{24}$ Agradecimientos: Se agradece al Consejo Nacional de Investigaciones Científicas y Técnicas (CONICET), la Universidad Nacional del Sur (UNS) y al proyecto COMET-LA (Gestión basada en la comunidad de los desafíos ambientales en América Latina) por el financiamiento del presente trabajo. Al Instituto Nacional de Tecnología Agropecuaria (INTA), al Instituto Argentino de Oceanografía (IADO) y a la UNS por el suministro de los datos meteorológicos. 